\title{
A CHARACTERIZATION OF BADLY APPROXIMABLE FUNCTIONS
}

\author{
BY
}

\section{S. J. POREDA}

ABSTRACT. Complex valued functions, continuous on a closed Jordan curve in the plane and having the property that all of their polynomials of best uniform approximation on that curve are identically zero are characterized in terms of their mapping properties on that curve.

1. Introductory remarks and definitions. For a continuous complex valued function $f$ defined on a compact set $E$ in the plane let

$$
\|f\|_{E}=\max _{z \in E}|f(z)| \text {. }
$$

Also for $n \in Z^{+}$let $p_{n}(f, E)$ denote the polynomial of degree $n$ of best uniform approximation to $f$ on $E$.

Now if $\Gamma$ is a closed Jordan curve and if $f$ is a continuous complex valued function defined on $\Gamma$ we then say that $f$ is badly approximable on $\Gamma$ if $p_{n}(f, \Gamma) \equiv 0$ for all $n \in Z^{+}$. For example, $f(z)=1 / z$ is badly approximable on the unit circle $U$.

It should be noted that if $f$ is not badly approximable on $\Gamma$ then $f$ cannot in general be uniformly approximated by polynomials on $\Gamma$. For example, if $f(z)=1 / z(1-a z)$, where $0<|a|<1$, then $f$ is not badly approximable on the unit circle $U$ but $\|f-p\|_{U}>1$ for any polynomial $p$.

The purpose of this paper is to draw a connection between the mapping properties of a continuous complex valued function $f$ on a closed Jordan curve $\Gamma$ and the approximability of $f$ on $\Gamma$. In doing so, it is hoped that some light will be shed on the problem of characterizing the behavior of the deviations; $\left[f-p_{n}(f, \Gamma)\right]$ as these functions appear more like a function that is badly approximable as $n$ increases.

2. Main theorem. Suppose $f$ is continuous and nonvanishing on the positive ly oriented Jordan curve $\Gamma$. Then let

$$
\Delta(f, \Gamma)=\frac{1}{2 \pi} \int_{\Gamma} d \operatorname{Arg} f(z) .
$$

$\Delta(f, \Gamma)$ is simply the winding number of the closed curve $f(\Gamma)$ with respect to

Received by the editors May 6, 1971 .

AMS 1969 subject classifications. Primary 4140; Secondary 3070.

Key words and phrases. Badly approximable functinns, best uniform approximation, closed Jordan curve. 
the origin. We can now state

Theorem 1. If $f$ is a continuous complex valued function defined on $\Gamma, a$ positively oriented Jordan curve, then $f$ is badly approximable on $\Gamma$ if and only if $|f|$ is identically constant on $\Gamma$ and if $\Delta(f, \Gamma)<0$.

Before proving Theorem 1 we will first examine its implications in the special case where $f$ is meromorphic inside $\Gamma$.

3. The meromorphic case. Suppose $f$ and $\Gamma$ are as before except that in addition we know that $f$ is the continuous extension of a function meromorphic interior to $\Gamma$. Let $\left\{\beta_{1}, \beta_{2}, \cdots, \beta_{n}\right\}$ be the poles and $\left\{\alpha_{1}, \alpha_{2}, \cdots, \alpha_{m}\right\}$ be the zeros of $f$ inside $\Gamma$ (counting multiplicities in each case). We then have

Corollary. $f$ is badly approximable on $\Gamma$ if and only if $f$ is of the form

$$
f(z)=K\left[\prod_{i=1}^{n}\left(\frac{1-\overline{\Phi\left(\beta_{i}\right)} \Phi(z)}{\Phi(z)-\Phi\left(\beta_{i}\right)}\right)\right] \cdot\left[\prod_{j=1}^{m}\left(\frac{\Phi(z)-\Phi\left(\alpha_{i}\right)}{1-\overline{\Phi\left(\alpha_{i}\right)} \Phi(z)}\right)\right],
$$

where $K$ is a nonzero constant, $m-n<0$ and $w=\Phi(z)$ conformally maps the interior of $\Gamma$ onto the open unit disc and maps $\Gamma$ continuously onto the unit circle $U$ in a one-to-one fashion. The existence of sucb a function is guaranteed by the extended Riemann mapping theorem.

Proof. Suppose $f$ is badly approximable on $\Gamma$. By Theorem 1 the function $f \circ \Phi^{-1}$ will then be continuous and of constant modulus $(>0)$ on the unit circle $U$ and meromorphic in the open unit disc having poles $\left\{\Phi\left(\beta_{1}\right), \Phi\left(\beta_{2}\right), \cdots, \Phi\left(\beta_{n}\right)\right\}$ and zeros $\left\{\Phi\left(\alpha_{1}\right), \Phi\left(\alpha_{2}\right), \cdots, \Phi\left(\alpha_{m}\right)\right\}$ there. As a consequence of Rouche's theorem, $f \circ \Phi^{-1}$ must then be of the form

$$
f \circ \Phi^{-1}(w)=K\left[\prod_{i=1}^{n}\left(\frac{1-\overline{\Phi\left(\beta_{i}\right)} w}{w-\Phi\left(\beta_{i}\right)}\right)\right] \cdot\left[\prod_{i=1}^{m}\left(\frac{w-\Phi\left(\alpha_{j}\right)}{\left.1-\overline{\Phi\left(\alpha_{j}\right)}\right)}\right)\right] .
$$

But $\Delta(f, \Gamma)=\Delta\left(f \circ \Phi^{-1}, U\right)=m-n$, so by Theorem $1, m-n<0$ if $f$ is badly approximable on $\Gamma$. The "sufficient" portion is immediate.

4. Preliminary results. Before proceeding with the proof of Theorem 1 we need use of several results which in themselves are interesting.

Theorem 2. If $f$ is continuous and of constant modulus on $\Gamma$, a closed Jordan curve, and if $\Delta(f, \Gamma)<0$, then $f$ is badly approximable.

Proof. By Kolmogorov's theorem [2, p. 15], $p_{n}(f, \Gamma) \equiv 0$ for all $n$ if and only if, for any polynomial $q, \min _{Z \in \Gamma} \operatorname{Re}[f(z) \overline{q(z)}] \leq 0$. If for some polynomial $q$ this does not hold, then $\operatorname{Re}[f(z) q(z)]>0$ for all $z \in \Gamma$. Thus as $z$ traverses $\Gamma$ once 
in a counterclockwise direction, the argument of the ratio $[f(z) / q(z)]$ has no net change, thus implying that the argument of $f(z)$ and the argument of $q(z)$ increase by the same net amount, namely $2 \pi \Delta(f, \Gamma)$, which is negative. This is clearly impossible since $q$ is a polynomial.

Theorem 3. If $f$ is continuous, not identically zero on $\Gamma$, a closed Jordan curve, and if $|f|$ is not constant on $\Gamma$, then $f$ is not badly approximable.

Proof. Since $|f|$ is not constant on $\Gamma$ there exists $z_{0} \in \Gamma$ such that $\left|f\left(z_{0}\right)\right|<\|f\|_{\Gamma}$. Since $f$ is continuous, there exists an open connected arc $\sigma \subset \Gamma$ containing $z_{0}$ such that, for $z \in \sigma,|f(z)|<\left[\|f\|_{\Gamma}-\epsilon\right]$ for some $\epsilon>0$. Thus $\Gamma-\sigma$ is a closed connected Jordan arc on which $f$ is continuous and so by Mergelyan's theorem [2, p. 3511 there exists a polynomial $q_{m}$ (of some degree $m$ ) such that

$$
\left\|f-q_{m}\right\|_{\Gamma-r}<\|f\|_{\Gamma}-\epsilon .
$$

If we choose $\lambda, 0<\lambda<1$, such that $\lambda\left\|q_{m}\right\|_{\Gamma}<\epsilon / 2$, we have

$$
\begin{aligned}
& \left\|f-\lambda q_{m}\right\|_{\Gamma-\sigma}<(1-\lambda)\|f\|_{\Gamma}+\lambda\left[\|f\|_{\Gamma}-\epsilon\right]=\|f\|_{\Gamma}-\lambda \epsilon \text {, and } \\
& \left\|f-\lambda q_{m}\right\|_{\sigma} \leq\|f\|_{\sigma}+\lambda\left\|_{m}\right\|_{\sigma} \leq\|f\|_{\Gamma}-\epsilon+\epsilon / 2=\|f\|_{\Gamma}-\epsilon / 2 .
\end{aligned}
$$

Thus $\left\|f-\lambda q_{m}\right\|_{\Gamma}<\|f\|_{\Gamma}$ and so $p_{m}(f, \Gamma) \not \equiv 0$.

Theorem 4. If $f$ is continuous, and nonvanisbing on the unit circle $U$ and if $\Delta(f, U) \geq 0$, then given any $\eta>0$ there exists a polynomial $p$ such that

$$
\cdot|\arg f(z)-\arg p(z)|<\eta \quad \text { for all } z \in U \text {. }
$$

The proof of Theorem 4 requires use of the following lemma.

Lemma. Suppose $R(z)$ is a rational function that neither vanishes nor bas a pole on $U$ and that has exactly $N$ zeros and $P$ poles (counting multiplicities) inside $U$. Then for all $\eta>0$ there exists a polynomial $p(z)$ that does not vanish on $U$ and sucb that

$$
|\arg R(z)-\arg p(z)|<\eta \quad \text { for all } z \in U
$$

if and only if $N-P \geq 0$.

Proof. Let

$$
R(z)=\left[K \prod_{i=1}^{N+m}\left(z-\alpha_{i}\right)\right] /\left[\prod_{j=1}^{P+l}\left(z-\beta_{j}\right)\right]
$$

where $K$ is a constant, $\left|\alpha_{i}\right|<1$ for $i=1, \cdots, N,\left|\alpha_{i}\right|>1$ for $i=N+1, \cdots$, $N+m,\left|\beta_{j}\right|>1$ for $j=1, \cdots, l$ and $\left|\beta_{j}\right|<1$ for $j=l+1, \cdots, P+l$. Now let 
$b_{\nu}=1 / \bar{a}_{\nu}$ for $\nu=1,2, \cdots, N$ and $b_{\nu+N}=\beta_{\nu}$ for $\nu=1,2, \cdots, l$. Note that $\left|b_{\nu}\right|>1$ for $\nu=1,2, \cdots, N+l$ so that we can choose $M \in Z^{+}$such that

$$
\left|b_{\nu}\right|^{M}>[\sin (\eta /[N+l])]^{-1} \text { for } \nu=1,2, \cdots, N+l \text {. }
$$

If we now assume that $N-P \geq 0$, and let $p(z)=L \Pi_{k=1}^{\lambda}\left(z-a_{k}\right)$ where $L$ is an appropriate constant and $\lambda=(N+l)(M-1)+m+N$, the "sufficient" portion of our lemma follows if we choose the $a_{k}$ 's in the following manner:

$$
\begin{aligned}
& a_{k}=1 / \bar{\beta}_{l+k} \text { for } k=1,2, \cdots, P \\
& a_{P+k}=a_{N+k} \quad \text { for } k=1,2, \cdots, m, \\
& a_{P+m+k}=0 \quad \text { for } k=1,2, \cdots, N-P \text {. }
\end{aligned}
$$

The $(N+l)(M-1)$ remaining zeros of $p(z)$, namely $\left\{a_{N+m+k}\right\}_{k=1}^{k=(N+l)(M-1)}$ are chosen so that

$$
\prod_{k=1}^{(N+l)(M-1)}\left(z-a_{N+m+k}\right)=\left[\prod_{\nu=1}^{N+l}\left(z^{M}-b_{\nu}^{M}\right)\right] /\left[\prod_{\nu=1}^{N+l}\left(z-b_{\nu}\right)\right] .
$$

In order to demonstrate this and using that for $|z|=1, \bar{z}=1 / z$, we have that

$$
\begin{aligned}
& {\left[\frac{p(z)}{|p(z)|} / \frac{R(z)}{|R(z)|}\right]^{2}=\left[\frac{p(z)}{\overline{p(z)}} \cdot \frac{R(z)}{\overline{R(z)}}\right]} \\
& =\left[\left(\frac{L}{\bar{L}}\right) \prod_{k=1}^{\lambda}\left(\frac{z-a_{k}}{1 / z-\bar{a}_{k}}\right)\right] \cdot\left[\left(\frac{\bar{K}}{K}\right) \prod_{i=1}^{N+m}\left(\frac{1 / z-\bar{a}_{i}}{z-a_{i}}\right) \prod_{j=1}^{P+l}\left(\frac{z-\beta_{j}}{1 / z-\bar{\beta}_{j}}\right)\right] .
\end{aligned}
$$

However,

$$
\prod_{k=1}^{N+m}\left(z-a_{k}\right)=z^{N-P}\left[\prod_{j=l+1}^{P+l}\left(z-1 / \bar{\beta}_{j}\right)\right] \cdot\left[\prod_{i=N+1}^{N+m}\left(z-\alpha_{i}\right)\right],
$$

and so

$$
\begin{aligned}
\prod_{k=1}^{N+m}\left(\frac{z-a_{k}}{1 / z-\bar{a}_{k}}\right) & =z^{2(N-P)+(m+P)}\left[\prod_{j=l+1}^{P+l}\left(\frac{z-1 / \bar{\beta}_{j}}{1-z / \beta_{j}}\right)\right] \cdot\left[\prod_{i=N+1}^{N+m}\left(\frac{z-\alpha_{i}}{1-\bar{\alpha}_{i} z}\right)\right] \\
& =z^{2 N+m-P} \prod_{j=l+1}^{P+l}\left(\frac{\beta_{j}}{\bar{\beta}_{j}}\right)\left[\prod_{i=l+1}^{P+l}\left(\frac{1-\bar{\beta}_{j} z}{z-\beta_{j}}\right)\right] \cdot\left[\prod_{i=N+1}^{N+m}\left(\frac{z-\alpha_{i}}{1-\bar{\alpha}_{i} z}\right)\right] .
\end{aligned}
$$


Therefore (1) can be written

$$
\left\{\left(\frac{L \bar{K}}{\bar{L} K}\right) \cdot\left[\prod_{j=l+1}^{P+l}\left(\frac{\beta_{j}}{\bar{\beta}_{j}}\right)\right] \cdot\left[z^{2 N} \prod_{k=N+m+1}^{\lambda}\left(\frac{z-a_{k}}{1 / z-\bar{a}_{k}}\right)\right]\right.
$$

$$
\left.\left[\prod_{i=1}^{N}\left(\frac{1 / z-\bar{\alpha}_{i}}{z-\alpha_{i}}\right)\right] \cdot\left[\prod_{j=1}^{l}\left(\frac{z-\beta_{j}}{1 / z-\bar{\beta}_{j}}\right)\right]\right\}
$$

But now recalling how we defined the $b_{\nu}$ 's we have

$$
\begin{gathered}
{\left[\prod_{i=1}^{N}\left(\frac{1 / z-\bar{\alpha}_{i}}{z-\alpha_{i}}\right)\right] \cdot\left[\prod_{j=1}^{l}\left(\frac{z-\beta_{j}}{1 / z-\bar{\beta}_{j}}\right)\right]} \\
=z^{l-N} \prod_{i=1}^{N}\left(\frac{\bar{\alpha}_{i}}{\alpha_{i}}\right)\left[\prod_{i=1}^{N}\left(\frac{z-1 / \bar{\alpha}_{i}}{1-z / \alpha_{i}}\right)\right] \cdot\left[\prod_{j=1}^{l}\left(\frac{z-\beta_{j}}{1-\bar{\beta}_{j} z}\right)\right] \\
=z^{l-N} \prod_{i=1}^{N}\left(\frac{\bar{\alpha}_{i}}{a_{i}}\right)\left[\prod_{\nu=1}^{N+l}\left(\frac{z-b_{\nu}}{1-\bar{b}_{\nu} z}\right)\right]
\end{gathered}
$$

and so, by our choice of the $a_{k}$ 's, $k=N+m+1, \cdots,(N+l)(M-1)+N+m$;

$$
\begin{gathered}
{\left[\prod_{k=N+m+1}^{\lambda}\left(\frac{z-a_{k}}{1 / z-\bar{a}_{k}}\right)\right] \cdot\left[\prod_{i=1}^{N}\left(\frac{1 / z-\bar{\alpha}_{i}}{z-\alpha_{i}}\right)\right] \cdot\left[\prod_{j=1}^{l}\left(\frac{z-\beta_{j}}{1 / z-\bar{\beta}_{j}}\right)\right]} \\
=\left[z^{(N+l)(M-1)} \prod_{k=N+m+1}^{\lambda}\left(\frac{z-\dot{a}_{k}}{1-\bar{a}_{k} z}\right)\right] \cdot\left[z^{l-N} \prod_{i=1}^{N}\left(\frac{\bar{\alpha}_{i}}{\alpha_{i}}\right)\right] \cdot\left[\prod_{\nu=1}^{N+l}\left(\frac{z-b_{\nu}}{1-\bar{b}_{\nu} z}\right)\right] \\
=\left[\prod_{i=1}^{N}\left(\frac{\bar{a}_{i}}{a_{i}}\right)\right] \cdot\left[z^{M(N+l)-2 N} \prod_{\nu=1}^{N+l}\left(\frac{z^{M}-b_{\nu}^{M}}{1-\bar{b}_{\nu}^{M} z^{M}}\right)\right] .
\end{gathered}
$$

It is now possible to express (2) as

$$
\left(\frac{L \bar{K}}{\bar{L} K}\right)\left[\prod_{i=l+1}^{P+l}\left(\frac{\beta_{j}}{\bar{\beta}_{j}}\right)\right] \cdot\left[\prod_{i=1}^{N}\left(\frac{\bar{\alpha}_{i}}{\alpha_{i}}\right)\right] \cdot\left[z^{M(N+l)} \prod_{\nu=1}^{N+l}\left(\frac{z^{M}-b_{\nu}^{M}}{1-\bar{b}_{\nu}^{M} z^{M}}\right)\right] .
$$

If we now choose

$$
L= \pm K\left[\prod_{j=l+1}^{P+l}\left(\beta_{j}^{-1}\right)\right] \cdot\left[\prod_{i=1}^{N} \alpha_{i}\right] \cdot\left[\prod_{\nu=1}^{N+l}\left(\frac{\bar{b}_{\nu}}{b_{\nu}}\right)^{M}\right]^{1 / 2}
$$


where the sign of $L$ is to be determined later; we will then have that

$$
\left[\frac{p(z)}{|p(z)|} / \frac{R(z)}{|R(z)|}\right]^{2}=\left[\prod_{\nu=1}^{N+l}\left(\frac{\bar{b}_{\nu}}{b_{\nu}}\right)^{M}\right] \cdot\left[z^{M(N+l)} \prod_{\nu=1}^{N+l}\left(\frac{z^{M}-b_{\nu}^{M}}{1-\bar{b}_{\nu}^{M} z^{M}}\right)\right] \text {. }
$$

But then since

$$
\begin{gathered}
\operatorname{Arg}\left[z^{M}\left(\frac{\bar{b}_{\nu}}{b_{\nu}}\right)^{M}\left(\frac{z^{M}-b_{\nu}^{M}}{1-\bar{b}_{\nu} z^{M}}\right)\right]=\operatorname{Arg}\left[\frac{1-z^{M} / b_{\nu}^{M}}{1-\bar{z}^{M} / \bar{b}_{\nu}^{M}}\right] \\
=2 \operatorname{Arg}\left[1-z^{M} / b_{\nu}^{M}\right], \text { where }-\pi<\operatorname{Arg} \omega \leq \pi,
\end{gathered}
$$

it follows that

$$
\begin{aligned}
\operatorname{Arg}\left[\frac{p(z)}{|p(z)|} / \frac{R(z)}{|R(z)|}\right]^{2}=2 \sum_{\nu=1}^{N+l} \operatorname{Arg}\left[1-z^{M} / b_{\nu}^{M}\right] \\
\leq 2 \sum_{\nu=1}^{N+l} \sin ^{-1}\left(\left|b_{\nu}\right|^{-M} ;<2 \sum_{\nu=1}^{N+l} \sin ^{-1}(\sin [\eta / N+l])=2 \eta .\right.
\end{aligned}
$$

Choosing the sign of $L$ appropriately we will then have

$$
\operatorname{Arg}\left[\frac{p(z)}{|p(z)|} / \frac{R(z)}{|R(z)|}\right]<\eta
$$

and so it follows that

$$
|\arg R(z)-\arg p(z)|<\eta \text { for all } z \in U
$$

where arg is a suitably chosen branch of the Arg function for each $z$.

The "necessary" portion of our lemma follows if we apply the argument used in proving Theorem 2.

We will now proceed with the proof of Theorem 4.

Proof. Since $f(z)$ is continuous and nonvanishing on $U$ by Weierstrass' theorem [2, p. 31 we have that given any $\eta_{1}>0$ there exists a rational function of the form $R(z)=\sum_{k=-n}^{k=n} a_{k} z^{k}$ such that

(i) $R(z)$ does not vanish on $U$,

(ii) $|\arg f(z)-\arg \dot{R}(z)|<\eta_{1}$ for all $z \in U$, and

(iii) $\Delta(R, U)=\Delta(f, U)$.

Since $\Delta(f, U) \geq 0$ it then follows that $N-P \geq 0$ where $N$ is the number of zeros of $R(z)$ inside $U$ and $P$ is the number of poles of $R(z)$ inside $U$.

Now by the previous lemma, given any $\eta_{2}>0$ there exists a polynomial 
$p(z)$ which does not vanish on $U$ and for which

$$
|\arg R(z)-\arg p(z)|<\eta_{2} \quad \text { for all } z \in U
$$

So choosing $\eta_{1}$ and $\eta_{2}$ such that $\eta_{1}+\eta_{2}<\eta$ our theorem thus follows.

5. Proof of main theorem. Let $f$ be continuous and of constant modulus $(>0)$ on $\Gamma$, a closed Jordan curve, and suppose $\Delta(f, \Gamma) \geq 0$. Also, let $D$ denote the domain bounded by $\Gamma$. As a consequence of the Riemann mapping theorem there exists a function $z=\Phi(w)$ which conformally maps $D$ onto the open unit disc $\{|z|<1\}$. Furthermore, $\Phi$ has a continuous extension to the boundary of $D$, namely $\Gamma$, and it maps $\Gamma$ onto the unit circle $U$ in a one-to-one fashion.

The function $f \circ \Phi^{-1}$ will then be continuous and of constant modulus on $U$ with $\Delta\left(f \circ \Phi^{-1}, U\right) \geq 0$. Thus given any $\eta_{1}>0$, by Theorem 4 there exists a polynomial $P_{n}$ (of some degree $n$ ) which does not vanish on $U$ and for which $\left|\arg f \circ \Phi^{-1}(z)-\arg P_{n}(z)\right|<\eta_{1}$ for all $z \in U$.

The function $\Phi$ is analytic in $D$, continuous in $D+\Gamma$, and so again by Mergelyan's theorem we have that given any $\epsilon_{1}>0$ there exists a polynomial $Q_{m}$ (of some degree $m$ ) for which $\left\|\Phi-Q_{m}\right\|_{\Gamma}<\epsilon_{1}$.

Since $P_{n}$ is uniformly continuous on any compact set, given any $\epsilon_{2}>0$, if $\epsilon_{1}$ is chosen sufficiently small we will have $\left\|P_{n} \circ \Phi-P_{n} \circ Q_{m}\right\|_{\Gamma}<\epsilon_{2^{*}}$. Furthermore, since $P_{n}$ does not vanish on $U$, given any $\eta_{2}>0$, by choosing $\epsilon_{2}$ sufficiently small we can get $\left|\arg P_{n} \circ \Phi(w)-\arg P_{n} \circ Q_{m}\right|<\eta_{2}$ for all $w \in \Gamma$.

Now by simply choosing $\eta_{1}, \eta_{2}<\pi / 4$ we will then have

$$
\begin{aligned}
& \left|\arg f(w)-\arg P_{n} \circ Q_{m}(w)\right|=\left|\arg f \circ \Phi^{-1}(z)-\arg P_{n} \circ Q_{m}(w)\right| \\
& \leq\left|\arg f \circ \Phi^{-1}(z)-\arg P_{n}(z)\right|+\left|\arg P_{n} \circ \Phi(w)-\arg P_{n} \circ Q_{m}(w)\right| \\
& <\pi / 4+\pi / 4=\pi / 2 \text { for all } w \in \Gamma .
\end{aligned}
$$

Hence $\operatorname{Re}\left[f(w) \overline{\left.P_{n} \circ Q_{m}(w)\right]}>0\right.$ for all $w \in \Gamma$. But since $P_{n} \circ Q_{m}$ is a polynomial (of some degree $n m$ ), we have by Kolomogorov's theorem [2, p. 15] that $p_{n m}(f, \Gamma) \not 0$. Theorem 1 now follows if we apply Theorems 2,3 , and 4 .

Since, in the above proof, $\eta_{1}$ and $\eta_{2}$ can be made arbitrarily small, we have the following

Corollary. If $f$ is continuous and of constant modulus $(>0)$ on $\Gamma$, a closed Jordan curve, and if $\Delta(f, \Gamma) \geq 0$, then given any $\eta>0$ there exists a polynomial $p_{n}$ for which $\left|\arg f(z)-\arg p_{n}(z)\right|<\eta$ for all $z \in \Gamma$. 


\section{REFERENCES}

1. A. I. Markuševič, Theory of functions of a complex variable. Vol. III, GITTL, Mosciow, 1950; English transl., Prentice-Hall, Englewood Cliffs, N. J., 1967. MR 12, 87; MR $35 \# 6799$.

2. J. L. Walsh, Interpolation and approximation by rational functions in the complex domain, 3rd ed., Amer. Math. Soc. Colloq. Publ., vol. 20, Amer. Math. Soc., Providence, R. I., 1960. MR $36 \# 1672$ a.

DEPARTMENT OF MATHEMATICS, CLARK UNIVERSITY, WORCESTER, MASSACHUSETTS 01610 